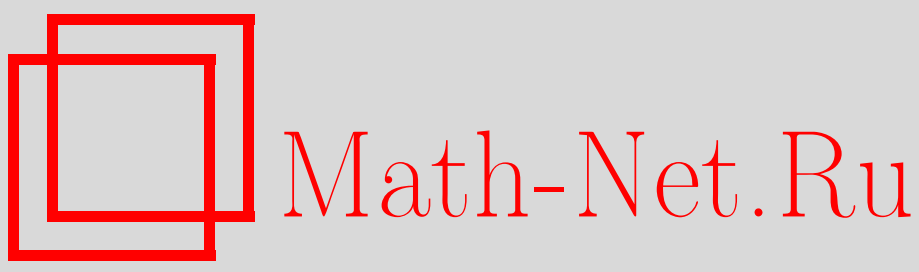

A. E. Kuvaev, Necessary conditions for the residual nilpotency of certain group theory constructions, Sibirsk. Mat. Zh., 2019, Volume 60, Number 6, 1335-1349

DOI: https://doi.org/10.33048/smzh.2019.60.612

Use of the all-Russian mathematical portal Math-Net.Ru implies that you have read and agreed to these terms of use http://www . mathnet.ru/eng/agreement

Download details:

IP: 54.198 .67 .100

April 26, 2023, 17:52:01

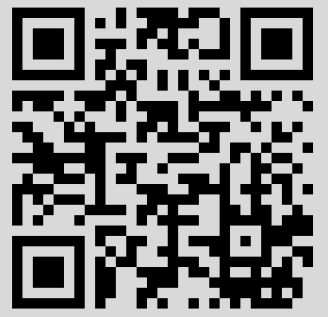


Сибирский математический журнал

Ноябрь-декабрь, 2019. Том 60, № 6

УДК 512.543

\title{
НЕОБХОДИМЫЕ УСЛОВИЯ НИЛЬПОТЕНТНОЙ АППРОКСИМИРУЕМОСТИ НЕКОТОРЫХ ТЕОРЕТИКО-ГРУППОВЫХ КОНСТРУКЦИЙ
}

\section{А. Е. Куваев}

\begin{abstract}
Аннотация. Пусть $G$ - граф групп, каждая вершинная группа графа $G$ локально удовлетворяет нетривиальному тождеству, каждая реберная подгруппа графа $G$ собственным образом содержится в соответствующих вершинных группах и имеет по крайней мере в одной из них индекс, больший 2. Доказано, что если фундаментальная группа $F$ графа $G$ локально аппроксимируется нильпотентными группами, то существует простое число $p$ такое, что каждая реберная подгруппа $p^{\prime}$-изолирована в соответствующих вершинных группах. Доказано также, что если $F-$ свободное произведение произвольного семейства групп с одной объединенной подгруппой или HNN-расширение с множеством проходных букв, то тот же результат имеет место и без ограничений на индексы реберных подгрупп.
\end{abstract}

DOI 10.33048/smzh.2019.60.612

Ключевые слова: фундаментальная группа графа групп, обобщенное свободное произведение, HNN-расширение, нильпотентная аппроксимируемость, аппроксимируемость конечными $p$-группами.

\section{$\S 1$. Введение}

Группу $X$ будем называть локально удовлетворяющей нетривиальному тождеству, если любая конечно порожденная подгруппа группы $X$ удовлетворяет нетривиальному тождеству (не обязательно одному и тому же для всех подгрупп). Будем говорить, что группа $X$ локально аппроксимируется нильпотентными группами, если любая ее конечно порожденная подгруппа $Y$ нильпотентно аппроксимируема, т. е. для каждого отличного от единицы элемента $y \in Y$ существует гомоморфизм $\sigma$ группы $Y$ на нильпотентную группу, причем $y \sigma \neq 1$. Отметим, что группа может не быть нильпотентно аппроксимируемой и не удовлетворять никакому нетривиальному тождеству, но обладать указанными свойствами локально (соответствующий пример приводится в конце статьи).

Целью данной работы является отыскание необходимых условий локальной аппроксимируемости нильпотентными группами для свободных конструкций, составленных из групп, локально удовлетворяющих нетривиальному тождеству. А именно, будет показано, что при определенных ограничениях таким условием является $p^{\prime}$-изолированность всех объединенных или связанных подгрупп для некоторого простого числа $p$ (напомним, что подгруппа $Y$ группы $X$

Работа выполнена при финансовой поддержке Ивановского государственного университета (договор № 7/01-04-18).

(c) 2019 Куваев А. Е. 
называется $p^{\prime}$-изолированной в этой группе, если для всякого $x \in X$ и для всякого простого числа $q \neq p$ из условия $x^{q} \in Y$ следует, что $x \in Y$ ).

Перейдем к определению рассматриваемых в работе теоретико-групповых конструкций.

Пусть $\Gamma$ - некоторый ориентированный граф и $\bar{\Gamma}-$ неориентированный граф, который получается из Г путем удаления ориентации ребер. Будем говорить, что граф $Г$ связен, если связным является граф $\bar{\Gamma}$ (т. е. любые две вершины последнего соединяет как минимум один путь). Точно так же будем считать граф Г аииклическим, если этим свойством обладает граф $\bar{\Gamma}$. Если $e$ - ребро графа $\Gamma$, то через $e(1)$ и $e(-1)$ будем обозначать вершины графа $\Gamma$, являющиеся соответственно началом и концом ребра $e$.

Пусть $G=(V, E)$ - произвольный непустой связный ориентированный граф с множеством вершин $V$ и совокупностью ребер $E$ (число вершин и ребер не обязано быть конечным, допускаются кратные ребра и петли). Сопоставив каждой вершине $v \in V$ некоторую группу $F_{v}$, каждому ребру $e \in E-$ группу $H_{e}$ и вложения $\varphi_{+e}: H_{e} \rightarrow F_{e(1)}, \varphi_{-e}: H_{e} \rightarrow F_{e(-1)}$, получим граф групп $\mathscr{G}$, соответствующий графу $G$.

Пусть $T=\left(V, E_{T}\right)$ - некоторое максимальное поддерево графа $G$ (т. е. связный ациклический подграф графа $G$, содержащий все его вершины). Фундаментальной группой графа групп $\mathscr{G}$ называется группа

$$
\begin{array}{r}
F=\left\langle * F_{v}, t_{f} ; H_{e} \varphi_{+e}=H_{e} \varphi_{-e}, t_{f}^{-1}\left(H_{f} \varphi_{+f}\right) t_{f}=H_{f} \varphi_{-f}\right. \\
\left.\left(v \in V, e \in E_{T}, f \in E \backslash E_{T}\right)\right\rangle,
\end{array}
$$

образующими которой являются образующие групп $F_{v}(v \in V)$ и буквы $t_{f}$ $\left(f \in E \backslash E_{T}\right)$, а определяющими соотношениями - соотношения групп $F_{v}$, а также всевозможные соотношения вида

$$
\begin{aligned}
h \varphi_{+e}=h \varphi_{-e} & \left(e \in E_{T}, h \in H_{e}\right), \\
t_{f}^{-1}\left(h \varphi_{+f}\right) t_{f}=h \varphi_{-f} & \left(f \in E \backslash E_{T}, h \in H_{f}\right) .
\end{aligned}
$$

Можно показать (см., например, [1, гл. 1, предложение 20]), что фундаментальная группа графа $\mathscr{G}$ не зависит от выбора дерева $T$.

Если граф $\mathscr{G}$ является деревом, то его фундаментальная группа $F$ называется древесным произведением групп $F_{v}(v \in V)$ с объединенными подгруппами $H_{\varepsilon e}(e \in E, \varepsilon= \pm 1)$. Если в дополнение к этому для любых двух ребер $e, f \in E$ и для любых чисел $\varepsilon, \delta \in\{1,-1\}$ из равенства $e(\varepsilon)=f(\delta)$ вытекает, что $H_{e} \varphi_{\varepsilon e}=H_{f} \varphi_{\delta f}$, то в группе $F$ все подгруппы $H_{e} \varphi_{\varepsilon e}(e \in E, \varepsilon= \pm 1)$ оказываются совпадающими. Поэтому группу $F$, удовлетворяющую указанному дополнительному условию, будем называть свободным произведением семейcтва групn $\left\{F_{v} \mid v \in V\right\}$ с одной объединенной подгруппой. Нетрудно показать, что получающаяся таким образом группа изоморфна свободному произведению семейства групп $\left\{F_{v} \mid v \in V\right\}$ с одной объединенной подгруппой, определенному в соответствии с [2].

Если граф $\mathscr{G}$ содержит одну вершину $v$ и по крайней мере одно ребро, то его фундаментальная группа $F$ имеет представление

$$
F=\left\langle F_{v}, t_{f} ; t_{f}^{-1}\left(H_{f} \varphi_{+f}\right) t_{f}=H_{f} \varphi_{-f}(f \in E)\right\rangle
$$

и называется HNN-расширением группы $F_{v}$ с семейством проходных букв $\left\{t_{f} \mid\right.$ $f \in E\}$.

Основным результатом настоящей работы служит 
Теорема 1. Пусть $F$ - фундаментальная группа графа групп вида (1), каждая группа $F_{v}(v \in V)$ локально удовлетворяет нетривиальному тождеству и для любых $e \in E, \varepsilon= \pm 1$ подгруппа $H_{e} \varphi_{\varepsilon e}$ содержится в группе $F_{e(\varepsilon)}$ собственным образом. Если группа $F$ локально аппроксимируется нильпотентными группами и для всякого ребра $e \in E$ хотя бы один из индексов $\left[F_{e(1)}: H_{e} \varphi_{+e}\right]$, $\left[F_{e(-1)}: H_{e} \varphi_{-e}\right]$ больше двух, то существует простое число $p$ такое, что для каждого ребра $e \in E$ и для каждого числа $\varepsilon= \pm 1$ подгруппа $H_{e} \varphi_{\varepsilon e} p^{\prime}$-изолирована в группе $F_{e(\varepsilon)}$.

Следствиями теоремы 1 являются приводимые далее теоремы 2 и 3 , обобщающие основные результаты работ [3-5]. Отметим, что в отличие от теоремы 1 в них уже нет дополнительного ограничения на индексы объединенных и связанных подгрупп.

Теорема 2. Пусть $F-$ свободное произведение семейства групп $\left\{F_{v} \mid v \in V\right\}$ c одной объединенной подгруппой $H$, все группы $F_{v}$ локально удовлетворяют нетривиальному тождеству и хотя бы в двух из них подгруппа $H$ содержится собственным образом. Если группа $F$ локально аппроксимируется нильпотентными группами, то существует простое число $p$ такое, что подгруппа $H$ $p^{\prime}$-изолирована в группе $F_{v}$ для любой вершины $v \in V$.

Теорема 3. Пусть $F-\mathrm{HNN}-$ расширение вида $(2)$, группа $F_{v}$ локально удовлетворяет нетривиальному тождеству и для любых $f \in E, \varepsilon= \pm 1$ подгруппа $H_{f} \varphi_{\varepsilon f}$ содержится в группе $F_{v}$ собственным образом. Если группа $F$ локально аппроксимируется нильпотентными группами, то существует простое число $p$ такое, что для каждого ребра $f \in E$ и для каждого числа $\varepsilon= \pm 1$ подгруппа $H_{f} \varphi_{\varepsilon f} p^{\prime}$-изолирована в группе $F_{v}$.

Если $F$ - свободное произведение семейства групп $\left\{F_{v} \mid v \in V\right\}$ с одной объединенной подгруппой $H$ и неравенство $H \neq F_{v}$ имеет место только для одной вершины $v \in V$, то $F=F_{v}$ и из локальной нильпотентной аппроксимируемости этой группы, разумеется, не следует $p^{\prime}$-изолированность в ней подгруппы $H$ ни для какого простого числа $p$. Таким образом, теорема 2 в этом случае оказывается неверна. Утверждение теоремы 3 также перестает быть справедливым, если хотя бы одна из связанных подгрупп $H_{f} \varphi_{\varepsilon f}(f \in E, \varepsilon= \pm 1)$ совпадает с базовой группой $F_{v}$; соответствующий пример приводится в [5].

Теоремы 1-3 в сочетании с полученным в $[6,7]$ описанием изоляторов подгрупп нильпотентных и нильпотентно аппроксимируемых групп определенного вида, а также многочисленными результатами об аппроксимируемости свободных конструкций групп конечными $p$-группами могут послужить основой для отыскания критериев нильпотентной аппроксимируемости некоторых обобщенных свободных произведений и HNN-расширений нильпотентных и нильпотентно аппроксимируемых групп.

\section{$\S 2$. Некоторые вспомогательные утверждения}

Предложение 1 [8, лемма 2]. Пусть группа $X$ удовлетворяет нетривиальному тождеству. Тогда $X$ удовлетворяет нетривиальному тождеству вида

$$
w\left(y, x_{1}, x_{2}\right)=w_{0}\left(x_{1}, x_{2}\right) y^{\varepsilon_{1}} w_{1}\left(x_{1}, x_{2}\right) \ldots y^{\varepsilon_{n}} w_{n}\left(x_{1}, x_{2}\right),
$$

где $n \geq 1, \varepsilon_{1}, \ldots, \varepsilon_{n}= \pm 1$ и $w_{0}\left(x_{1}, x_{2}\right), \ldots, w_{n}\left(x_{1}, x_{2}\right) \in\left\{x_{1}^{ \pm 1}, x_{2}^{ \pm 1},\left(x_{1} x_{2}^{-1}\right)^{ \pm 1}\right\}$. 
Предложение 2. Пусть $p$ и $q$ - взаимно простые числа, $X-$ конечная группа порядка $p$. Тогда для любого элемента $x \in X$ найдется целое число $m$ такое, что $x=x^{q m}$.

Доказательство. Пусть $x \in X$. Тогда $x^{p}=1$. Так как $(q, p)=1$, существуют числа $m, k \in \mathbb{Z}$, удовлетворяющие условию $q m+p k=1$. Тогда $x=x^{q m+p k}=\left(x^{q}\right)^{m}\left(x^{p}\right)^{k}=x^{q m}$, что и требовалось.

Предложение 3. Пусть $X-$ некоторая группа, $Y$ и $Z-$ конечно порожденные подгруппы группы $X, x_{1} \in Y$ и $x_{2} \in Z$ - элементы такие, что $\left[x_{1}, x_{2}\right] \neq 1, p$ и $q-$ простые числа. Пусть также

$(a)$ для любого простого числа $r$ и для любого гомоморфизма $\rho$ подгруппы $Y$ на конечную $r$-группу из соотношения $r \neq p$ следует, что $x_{1} \rho=1$;

(b) для любого простого числа $s$ и для любого гомоморфизма $\sigma$ подгруппы $Z$ на конечную $s$-группу из соотношения $s \neq q$ следует, что $x_{2} \sigma=1$.

Если $p \neq q$, то группа $X$ не является локально аппроксимируемой нильпотентными группами.

ДокАЗАтЕЛЬство. Пусть $M$ и $N$ - конечные порождающие множества подгрупп $Y$ и $Z$ соответственно, $U$ - подгруппа, порожденная множеством $M \cup N$. Предположим, что $p \neq q$, но группа $X$ локально аппроксимируется нильпотентными группами. Тогда подгруппа $U$ аппроксимируется конечно порожденными нильпотентными группами. По теореме Гирша [9] всякая конечно порожденная нильпотентная группа финитно аппроксимируема. Поэтому подгруппа $U$ аппроксимируется конечными нильпотентными группами.

Пусть $x=\left[x_{1}, x_{2}\right], \tau$ - гомоморфизм подгруппы $U$ на конечную нильпотентную группу $Z$, переводящий $x$ в неединичный элемент, $t-$ простое число, делящее порядок элемента $x \tau$. По теореме Бернсайда - Виландта (см., например, [10, теорема 2.7]) группа $Z$ раскладывается в прямое произведение своих силовских подгрупп. Пусть $T$ - произведение всех силовских подгрупп группы $Z$, кроме подгруппы, соответствующей числу $t$. Тогда $x \tau \notin T$, поэтому композиция $\tau$ и естественного гомоморфизма группы $Z$ на фактор-группу $Z / T$ отображает $x$ в неединичный элемент конечной $t$-группы $Z / T$.

Если $t=q$, то согласно неравенству $p \neq q$ и условию $(a)$ элемент $x_{1}$ переходит в единицу под действием ограничения гомоморфизма $\tau$ на подгруппу $Y$. Если $t \neq q$, то согласно условию $(b)$ справедливо равенство $x_{2} \tau=1$. Так или иначе, но $x \tau=1$, что противоречит выбору гомоморфизма $\tau$.

\section{$\S 3$. Некоторые свойства обобщенных свободных произведений групп}

Пусть до конца этого раздела $F$ обозначает свободное произведение групп $A$ и $B$ с объединенной подгруппой $H$.

Запись элемента $f \in F$ в виде $f=f_{1} f_{2} \ldots f_{n}(n \geq 1)$ называется несократимой, если каждый элемент $f_{k}(1 \leq k \leq n)$ лежит в одном из множителей $A, B$, причем соседние элементы $f_{k}, f_{k+1}$ не лежат в одном и том же свободном множителе. Число $n$ называется длиной данной несократимой записи.

Приводимое далее предложение 4 является непосредственным следствием теоремы о нормальной форме для обобщенных свободных произведений двух групп (см., например, [11, следствие 4.4.1]). 
Предложение 4. Всякий элемент $f \in F$, обладающий хотя бы одной несократимой записью длины, большей 1 , не лежит ни в одном из свободных множителей $A, B$ и, в частности, отличен от единицы.

Предложение 5 [11, следствие 4.4.3]. В группе $F$ имеет место равенство $A \cap B=H$.

Предложение 6. Пусть группы $A$ и $B$ локально удовлетворяют нетривиальному тождеству, подгруппа $H$ содержится в каждой из них собственным образом и хотя бы один из индексов $[A: H],[B: H]$ больше двух. Пусть также существует элемент $a \in A \backslash H$ такой, что $a^{q} \in H$ для некоторого простого числа q. Тогда найдутся конечно порожденная подгруппа $S \leq F$ и элементы $g_{1}, g_{2} \in S$, обладающие следующими свойствами:

(a) элементы $g_{1}$ и $g_{2}$ имеют несократимые записи неединичной длины; при этом первый и последний слоги указанной записи элемента $g_{1}$ принадлежат множеству $A \backslash H$, а первый и последний слоги записи элемента $g_{2}-$ множеству $B \backslash H$;

$(b)$ для любого простого числа $p$ и для любого гомоморфизма $\sigma$ подгруппы $S$ на конечную $p$-группу из соотношения $p \neq q$ следует, что $g_{1} \sigma=g_{2} \sigma=1$.

ДокАЗАТЕЛЬСТво. Рассмотрим три взаимоисключающих случая:

1) $[B: H]>2$,

2) $[B: H]=2$ и $q>2$,

3) $[B: H]=2$ и $q=2$.

СлучАй $1 .[B: H]>2$. Пусть элементы $1, b_{1}, b_{2}$ являются представителями различных правых смежных классов группы $B$ по подгруппе $H$. Будучи конечно порожденной подгруппой группы $B$, группа $U=\operatorname{sgp}\left\{a^{q}, b_{1}, b_{2}\right\}$ удовлетворяет нетривиальному тождеству, которое в силу предложения 1 можно считать имеющим вид (3). Положим

$$
\begin{gathered}
S=\operatorname{sgp}\left\{a, b_{1}, b_{2}\right\}, \\
g_{1}=a^{-1} w\left(a, b_{1}, b_{2}\right) a=a^{-1} w_{0}\left(b_{1}, b_{2}\right) a^{\varepsilon_{1}} w_{1}\left(b_{1}, b_{2}\right) \ldots a^{\varepsilon_{n}} w_{n}\left(b_{1}, b_{2}\right) a, \\
g_{2}=w\left(a, b_{1}, b_{2}\right)=w_{0}\left(b_{1}, b_{2}\right) a^{\varepsilon_{1}} w_{1}\left(b_{1}, b_{2}\right) \ldots a^{\varepsilon_{n}} w_{n}\left(b_{1}, b_{2}\right) .
\end{gathered}
$$

Согласно предложению 1 элемент $w_{r}\left(b_{1}, b_{2}\right), r \in\{0, \ldots, n\}$, имеет вид $b_{1}^{ \pm 1}$ или $b_{2}^{ \pm 1}$, или $\left(b_{1} b_{2}^{-1}\right)^{ \pm 1}$. Так как элементы $b_{1}, b_{2}$ принадлежат разным правым смежным классам группы $B$ по подгруппе $H$ и отличны от единицы, то $w_{r}\left(b_{1}, b_{2}\right) \in B \backslash H$ для всякого $r \in\{0, \ldots, n\}$. Также по условию предложения справедливо соотношение $a \in A \backslash H$. Поэтому приведенные выше записи элементов $g_{1}, g_{2}$ несократимы и обладают свойством $(a)$.

Пусть $\sigma$ - некоторый гомоморфизм подгруппы $S$ на конечную $p$-группу и $p \neq q$. Из включения $a^{q} \in U$ и предложения 2 вытекает, что $a \sigma \in U \sigma$. Поскольку группа $U$ удовлетворяет тождеству (3), отсюда следует, что $g_{1} \sigma=$ $g_{2} \sigma=1$. Таким образом, для подгруппы $S$ и элементов $g_{1}, g_{2}$ выполняется и свойство $(b)$.

Случай 2. $[B: H]=2$ и $q>2$. Пусть $b \in B \backslash H$. Группа $U=\operatorname{sgp}\left\{a^{q}, b\right\}$ является конечно порожденной подгруппой группы $B$ и потому удовлетворяет нетривиальному тождеству, которое, как и выше, можно считать имеющим 
вид (3). Положим

$$
\begin{gathered}
S=\operatorname{sgp}\{a, b\} \\
g_{1}=w\left(b, a, a^{2}\right)=w_{0}\left(a, a^{2}\right) b^{\varepsilon_{1}} w_{1}\left(a, a^{2}\right) \ldots b^{\varepsilon_{n}} w_{n}\left(a, a^{2}\right), \\
g_{2}=b^{-1} w\left(b, a, a^{2}\right) b=b^{-1} w_{0}\left(a, a^{2}\right) b^{\varepsilon_{1}} w_{1}\left(a, a^{2}\right) \ldots b^{\varepsilon_{n}} w_{n}\left(a, a^{2}\right) b .
\end{gathered}
$$

Согласно предложению 1 для каждого $r \in\{0, \ldots, n\}$ справедливо включение $w_{r}\left(a, a^{2}\right) \in\left\{a^{ \pm 1}, a^{ \pm 2}\right\}$. По условию предложения $a \in A \backslash H$, а из неравенства $q>2$ и простоты числа $q$ вытекает также, что $a^{2} \in A \backslash H$. Следовательно, $w_{r}\left(a, a^{2}\right) \in A \backslash H$ для всякого $r \in\{0, \ldots, n\}$. Таким образом, записи элементов $g_{1}, g_{2}$ несократимы и обладают свойством $(a)$. Выполнение свойства $(b)$ проверяется так же, как и в случае 1.

СлучАй 3. $[B: H]=2$ и $q=2$. Пусть $b \in B \backslash H$. Из условия предложения и из того, что $[B: H]=2$, следует, что $[A: H]>2$. Пусть элементы $1, a_{1}$, $a_{2}$ являются представителями различных правых смежных классов группы $A$ по подгруппе $H$.

Так как $[B: H]=2$, то $b^{2} \in H$ и потому $U=\operatorname{sgp}\left\{b^{2}, a_{1}, a_{2}\right\}-$ конечно порожденная подгруппа группы $A$. Значит, она удовлетворяет нетривиальному тождеству, которое снова можно считать имеющим вид (3). Положим

$$
\begin{gathered}
S=\operatorname{sgp}\left\{a_{1}, a_{2}, b\right\}, \\
g_{1}=w\left(b, a_{1}, a_{2}\right)=w_{0}\left(a_{1}, a_{2}\right) b^{\varepsilon_{1}} w_{1}\left(a_{1}, a_{2}\right) \ldots b^{\varepsilon_{n}} w_{n}\left(a_{1}, a_{2}\right), \\
g_{2}=b^{-1} w\left(b, a_{1}, a_{2}\right) b=b^{-1} w_{0}\left(a_{1}, a_{2}\right) b^{\varepsilon_{1}} w_{1}\left(a_{1}, a_{2}\right) \ldots b^{\varepsilon_{n}} w_{n}\left(a_{1}, a_{2}\right) b .
\end{gathered}
$$

Как и в случае 1 , проверяется, что $w_{r}\left(a_{1}, a_{2}\right) \in A \backslash H$ для всякого $r \in$ $\{0, \ldots, n\}$. Также $b \in B \backslash H$. Поэтому приведенные выше записи элементов $g_{1}$, $g_{2}$ несократимы и обладают свойством $(a)$.

Пусть $\sigma$ - некоторый гомоморфизм подгруппы $S$ на конечную $p$-группу и $p \neq q$. Из соотношения $q=2$, включения $b^{2} \in U$ и предложения 2 вытекает, что $b \sigma \in U \sigma$. Поскольку группа $U$ удовлетворяет тождеству (3), отсюда следует, что $g_{1} \sigma=g_{2} \sigma=1$ и, значит, свойство $(b)$ также имеет место.

\section{$\S$ 4. Некоторые свойства HNN-расширений групп}

Пусть $F-\mathrm{HNN}$-расширение вида (2). Запись элемента $g \in F$ в виде

$$
g=g_{0} t_{f_{1}}^{\varepsilon_{1}} g_{1} \ldots g_{n-1} t_{f_{n}}^{\varepsilon_{n}} g_{n},
$$

где $g_{0}, \ldots, g_{n} \in F_{v}, f_{1}, \ldots, f_{n} \in E, \varepsilon_{1}, \ldots, \varepsilon_{n}= \pm 1$ и $n \geq 0$, называется приведенной, если в ней не встречаются подряд элементы $t_{f}^{-\varepsilon}, h, t_{f}^{\varepsilon}$, где $f \in E, \varepsilon= \pm 1$, $h \in H_{\varepsilon f}$. Число $n$ называется длиной данной приведенной записи. Следующее утверждение может быть выведено из леммы Бриттона для HNN-расширений с одной проходной буквой (см., например, $[12$, гл. IV, § 2]).

Предложение 7. Пусть $F-\mathrm{HNN}$-расширение вида (2). Всякий элемент $g \in F$, обладающий хотя бы одной приведенной записью длины, большей 0 , не принадлежит базовой группе $F_{v}$ и, в частности, отличен от 1.

Предложение 8. Пусть $F-\mathrm{HNN}$-расширение вида (2), причем граф $G$ содержит только одно ребро е. Пусть также подгруппа $H_{e} \varphi_{+e}$ собственным образом содержится в некоторой подгруппе $A$ группы $F_{v}$, локально удовлетворяющей нетривиальному тождеству, подгруппа $H_{e} \varphi_{-e}$ собственным образом содержится в некоторой подгруппе $B$ группы $F_{v}$, локально удовлетворяющей нетривиальному тождеству, и хотя бы один из индексов $\left[A: H_{e} \varphi_{+e}\right],\left[B: H_{e} \varphi_{-e}\right]$ 
больше двух. Если существуют простое число $q$ и элемент $a \in A \backslash H_{e} \varphi_{+e}$ такой, что $a^{q} \in H_{e} \varphi_{+e}$, или элемент $b \in B \backslash H_{e} \varphi_{-e}$ такой, что $b^{q} \in H_{e} \varphi_{-e}$, то найдутся конечно порожденная подгруппа $S \leq F$ и элементы $g_{1}, g_{2} \in S$, обладающие следующими свойствами:

(a) элементы $g_{1}$ и $g_{2}$ имеют приведенные записи ненулевой длины, при этом запись элемента $g_{1}$ начинается на $t_{e}^{-1}$ и заканчивается на $t_{e}$, а запись элемента $g_{2}$ начинается на $t_{e}$ и заканчивается на $t_{e}^{-1}$;

$(b)$ для любого простого числа $p$ и для любого гомоморфизма $\sigma$ подгруппы $S$ на конечную $p$-группу из соотношения $p \neq q$ следует, что $g_{1} \sigma=g_{2} \sigma=1$.

ДокАЗАТЕЛЬСТво. Поменяв, если необходимо, направление ребра $e$ на противоположное и подгруппы $A$ и $B$ местами, можно считать далее, что существует элемент $a \in A \backslash H_{e} \varphi_{+e}$ такой, что $a^{q} \in H_{e} \varphi_{+e}$. Рассмотрим три взаимоисключающих случая:

1) $\left[B: H_{e} \varphi_{-e}\right]>2$,

2) $\left[B: H_{e} \varphi_{-e}\right]=2$ и $q>2$,

3) $\left[B: H_{e} \varphi_{-e}\right]=2$ и $q=2$.

СлучАЙ 1. $\left[B: H_{e} \varphi_{-e}\right]>2$. Пусть элементы $1, b_{1}, b_{2}$ являются представителями различных правых смежных классов группы $B$ по подгруппе $H_{e} \varphi_{-e}$. Будучи конечно порожденной подгруппой группы $B$, группа $U=s g p\left\{t_{e}^{-1} a^{q} t_{e}, b_{1}, b_{2}\right\}$ удовлетворяет нетривиальному тождеству, которое в силу предложения 1 можно считать имеющим вид (3). Положим

$$
\begin{aligned}
S & =\operatorname{sgp}\left\{a, b_{1}, b_{2}, t_{e}\right\}, \\
g_{1} & =t_{e}^{-1} w\left(t_{e}^{-1} a t_{e}, b_{1}, b_{2}\right) t_{e} \\
& =t_{e}^{-1} w_{0}\left(b_{1}, b_{2}\right) t_{e}^{-1} a^{\varepsilon_{1}} t_{e} w_{1}\left(b_{1}, b_{2}\right) \ldots t_{e}^{-1} a^{\varepsilon_{n}} t_{e} w_{n}\left(b_{1}, b_{2}\right) t_{e}, \\
g_{2} & =t_{e} w\left(t_{e}^{-1} a t_{e}, b_{1}, b_{2}\right) t_{e}^{-1} \\
& =t_{e} w_{0}\left(b_{1}, b_{2}\right) t_{e}^{-1} a^{\varepsilon_{1}} t_{e} w_{1}\left(b_{1}, b_{2}\right) \ldots t_{e}^{-1} a^{\varepsilon_{n}} t_{e} w_{n}\left(b_{1}, b_{2}\right) t_{e}^{-1} .
\end{aligned}
$$

Как и при доказательстве предложения 6 , устанавливается, что $w_{r}\left(b_{1}, b_{2}\right) \in$ $B \backslash H_{e} \varphi_{-e}$ для всякого $r \in\{0, \ldots, n\}$. Отсюда и из соотношения $a \in A \backslash H_{e} \varphi_{+e}$ следует, что записи элементов $g_{1}, g_{2}$ являются приведенными и обладают свойством $(a)$.

Пусть $\sigma$ - некоторый гомоморфизм подгруппы $S$ на конечную $p$-группу и $p \neq q$. Из включения $t_{e}^{-1} a^{q} t_{e} \in U$ и предложения 2 вытекает, что $\left(t_{e}^{-1} a t_{e}\right) \sigma \in$ $U \sigma$. Поскольку группа $U$ удовлетворяет тождеству (3), отсюда следует, что $\left(t_{e} g_{1} t_{e}^{-1}\right) \sigma=\left(t_{e}^{-1} g_{2} t_{e}\right) \sigma=1$. Таким образом, для подгруппы $S$ и элементов $g_{1}$, $g_{2}$ выполняется и свойство $(b)$.

Случай 2. $\left[B: H_{e} \varphi_{-e}\right]=2$ и $q>2$. Пусть $b \in B \backslash H_{e} \varphi_{-e}$. Группа $U=$ $\operatorname{sgp}\left\{t_{e}^{-1} a^{q} t_{e}, b\right\}$ является конечно порожденной подгруппой группы $B$ и потому удовлетворяет нетривиальному тождеству, которое, как и выше, можно считать имеющим вид (3). Положим

$$
\begin{aligned}
S & =\operatorname{sgp}\left\{a, b, t_{e}\right\}, \\
g_{1} & =w\left(b, t_{e}^{-1} a t_{e}, t_{e}^{-1} a^{2} t_{e}\right) \\
& =w_{0}\left(t_{e}^{-1} a t_{e}, t_{e}^{-1} a^{2} t_{e}\right) b^{\varepsilon_{1}} w_{1}\left(t_{e}^{-1} a t_{e}, t_{e}^{-1} a^{2} t_{e}\right) \ldots b^{\varepsilon_{n}} w_{n}\left(t_{e}^{-1} a t_{e}, t_{e}^{-1} a^{2} t_{e}\right), \\
g_{2} & =t_{e}^{2} w\left(b, t_{e}^{-1} a t_{e}, t_{e}^{-1} a^{2} t_{e}\right) t_{e}^{-2} \\
& =t_{e}^{2} w_{0}\left(t_{e}^{-1} a t_{e}, t_{e}^{-1} a^{2} t_{e}\right) b^{\varepsilon_{1}} w_{1}\left(t_{e}^{-1} a t_{e}, t_{e}^{-1} a^{2} t_{e}\right) \ldots b^{\varepsilon_{n}} w_{n}\left(t_{e}^{-1} a t_{e}, t_{e}^{-1} a^{2} t_{e}\right) t_{e}^{-2} .
\end{aligned}
$$


Согласно предложению 1 для каждого $r \in\{0, \ldots, n\}$ справедливо включение

$$
w_{r}\left(t_{e}^{-1} a t_{e}, t_{e}^{-1} a^{2} t_{e}\right) \in\left\{t_{e}^{-1} a^{ \pm 1} t_{e}, t_{e}^{-1} a^{ \pm 2} t_{e}\right\} .
$$

Из соотношения $a \in A \backslash H_{e} \varphi_{+e}$, неравенства $q>2$ и простоты числа $q$ вытекает, что $a^{2} \in A \backslash H_{e} \varphi_{+e}$. Поэтому записи элементов $g_{1}, g_{2}$ являются приведенными и обладают свойством $(a)$. Выполнение свойства $(b)$ проверяется так же, как и в случае 1.

СлУчАЙ 3. $\left[B: H_{e} \varphi_{-e}\right]=2$ и $q=2$. Из условия предложения и равенства $\left[B: H_{e} \varphi_{-e}\right]=2$ следует, что $\left[A: H_{e} \varphi_{+e}\right]>2$. Пусть элементы $1, a_{1}, a_{2}$ являются представителями различных правых смежных классов группы $A$ по подгруппе $H_{e} \varphi_{+e}$. Пусть также $b \in B \backslash H_{e} \varphi_{-e}$. Так как $\left[B: H_{e} \varphi_{-e}\right]=2$, подгруппа $H_{e} \varphi_{-e}$ нормальна в $B$. Поэтому $b^{-1}\left(t_{e}^{-1} a^{q} t_{e}\right) b \in H_{e} \varphi_{-e}$ и $t_{e} b^{-1}\left(t_{e}^{-1} a^{q} t_{e}\right) b t_{e}^{-1} \in$ $H_{e} \varphi_{+e}$. Следовательно, группа $U=\operatorname{sgp}\left\{t_{e} b^{-1} t_{e}^{-1} a^{q} t_{e} b t_{e}^{-1}, a_{1}, a_{2}\right\}$ является конечно порожденной подгруппой группы $A$ и потому удовлетворяет нетривиальному тождеству, которое снова можно считать имеющим вид (3). Положим

$$
\begin{aligned}
S & =\operatorname{sgp}\left\{a, b, a_{1}, a_{2}, t_{e}\right\} \\
g_{1} & =t_{e}^{-1} w\left(t_{e} b^{-1} t_{e}^{-1} a t_{e} b t_{e}^{-1}, a_{1}, a_{2}\right) t_{e} \\
& =t_{e}^{-1} w_{0}\left(a_{1}, a_{2}\right) t_{e} b^{-1} t_{e}^{-1} a^{\varepsilon_{1}} t_{e} b t_{e}^{-1} w_{1}\left(a_{1}, a_{2}\right) \ldots t_{e} b^{-1} t_{e}^{-1} a^{\varepsilon_{n}} t_{e} b t_{e}^{-1} w_{n}\left(a_{1}, a_{2}\right) t_{e} \\
g_{2} & =t_{e} w\left(t_{e} b^{-1} t_{e}^{-1} a t_{e} b t_{e}^{-1}, a_{1}, a_{2}\right) t_{e}^{-1} \\
& =t_{e} w_{0}\left(a_{1}, a_{2}\right) t_{e} b^{-1} t_{e}^{-1} a^{\varepsilon_{1}} t_{e} b t_{e}^{-1} w_{1}\left(a_{1}, a_{2}\right) \ldots t_{e} b^{-1} t_{e}^{-1} a^{\varepsilon_{n}} t_{e} b t_{e}^{-1} w_{n}\left(a_{1}, a_{2}\right) t_{e}^{-1} .
\end{aligned}
$$

Как и выше, устанавливается, что $w_{r}\left(a_{1}, a_{2}\right) \in A \backslash H_{e} \varphi+e$ для всякого $r \in\{0, \ldots, n\}$. Кроме того, $a \in A \backslash H_{e} \varphi_{+e}, b \in B \backslash H_{e} \varphi_{-e}$. Поэтому записи элементов $g_{1}, g_{2}$ являются приведенными и обладают свойством $(a)$. Выполнение свойства $(b)$ проверяется так же, как и в случае 1 , с той лишь разницей, что вместо элементов $t_{e}^{-1} a t_{e}$ и $t_{e}^{-1} a^{q} t_{e}$ нужно рассмотреть элементы $t_{e} b^{-1}\left(t_{e}^{-1} a t_{e}\right) b t_{e}^{-1}$ и $t_{e} b^{-1}\left(t_{e}^{-1} a^{q} t_{e}\right) b t_{e}^{-1}$ соответственно.

\section{$\S 5$. Некоторые свойства фундаментальных групп произвольных графов групп}

Пусть $F-$ фундаментальная группа графа групп вида $(1)$ и $P$ - древесное произведение, соответствующее максимальному поддереву $T$. Тогда группа $F$ представляет собой HNN-расширение группы $P$ с семейством проходных букв $\left\{t_{f} \mid f \in E \backslash E_{T}\right\}$ и в силу теоремы 2 из [13] $P$ можно считать подгруппой в $F$. Если $e \in E \backslash E_{T}$ - некоторое ребро, то группа $F$ является и $\mathrm{HNN}$-расширением группы

$$
P(e)=\left\langle P, t_{e} ; t_{e}^{-1}\left(H_{e} \varphi_{+e}\right) t_{e}=H_{e} \varphi_{-e}\right\rangle
$$

с семейством проходных букв $\left\{t_{f} \mid f \in E \backslash\left(E_{T} \cup\{e\}\right)\right\}$. Следовательно, HNN-pacширение $P(e)$ также оказывается подгруппой в $F$. Наконец, если $\widetilde{T}=(\widetilde{V}, \widetilde{E})-$ поддерево дерева $T$, вершинам и ребрам которого сопоставлены те же группы и отображения, а $\widetilde{P}$ - соответствующее $\widetilde{T}$ древесное произведение, то согласно теореме 1 из [14] тождественное отображение образующих группы $\widetilde{P}$ в группу $P$ задает изоморфное вложение и, значит, группу $\widetilde{P}$ также можно считать подгруппой в $F$. Приведенные соображения позволяют применять к указанным выше подгруппам группы $F$ предложения 6,8 , и в доказательстве приводимого далее предложения 9 эта возможность будет использоваться без дополнительных оговорок. 
Предложение 9. Пусть $F-$ фундаментальная группа графа групп вида (1), каждая группа $F_{v}(v \in V)$ локально удовлетворяет нетривиальному тождеству и для любых $e \in E, \varepsilon= \pm 1$ подгруппа $H_{e} \varphi_{\varepsilon e}$ содержится в группе $F_{e(\varepsilon)}$ собственным образом. Пусть также группа $F$ локально аппроксимируется нильпотентными группами и для всякого ребра $e \in E$ хотя бы один из индексов $\left[F_{e(1)}: H_{e} \varphi_{+e}\right],\left[F_{e(-1)}: H_{e} \varphi_{-e}\right]$ больше двух. Если существуют (не обязательно различные) ребра $e, f \in E$, (не обязательно различные) числа $\varepsilon, \delta= \pm 1$, элементы $x_{e} \in F_{e(\varepsilon)} \backslash H_{e} \varphi_{\varepsilon e}, x_{f} \in F_{f(\delta)} \backslash H_{f} \varphi_{\delta f}$ и простые числа $p, q$ такие, что $x_{e}^{p} \in H_{e} \varphi_{\varepsilon e}, x_{f}^{q} \in H_{f} \varphi_{\delta f}$, то $p=q$.

ДокАЗАТЕЛЬСТво. Рассмотрим три взаимоисключающих случая:

1) $e, f \in E_{T}$;

2) $e \in E_{T}, f \notin E_{T}$ или $e \notin E_{T}, f \in E_{T}$;

3) $e, f \notin E_{T}$.

Случай 1. $e, f \in E_{T}$. Среди вершин $e(1), e(-1), f(1), f(-1)$ имеется по крайней мере две различные. Очевидно, что при изменении направлений любого числа ребер из множества $E_{T}$ представление группы $F$ остается тем же. Поэтому без потери общности можно считать, что $e(-1) \neq f(1)$, в дереве $T$ имеется путь, ведущий из вершины $f(1)$ в вершину $e(-1)$, и этот путь проходит через вершины $e(1)$ и $f(-1)$.

Согласно предложению 6 , применяемому сначала к обобщенному свободному произведению

$$
P_{e}=\left\langle F_{e(1)} * F_{e(-1)} ; H_{e} \varphi_{+e}=H_{e} \varphi_{-e}\right\rangle
$$

и элементу $x_{e}$, а затем к обобщенному свободному произведению

$$
P_{f}=\left\langle F_{f(1)} * F_{f(-1)} ; H_{f} \varphi_{+f}=H_{f} \varphi_{-f}\right\rangle
$$

и элементу $x_{f}$, существуют конечно порожденные подгруппы $S_{1} \leq P_{e}, S_{2} \leq P_{f}$ и элементы $g_{1} \in S_{1}, g_{2} \in S_{2}$, обладающие следующими свойствами:

$(a)$ элементы $g_{1}$ и $g_{2}$ имеют в группах $P_{e}$ и $P_{f}$ соответственно несократимые записи неединичной длины, при этом первый и последний слоги указанной записи элемента $g_{1}$ принадлежат множеству $F_{e(-1)} \backslash H_{e} \varphi_{-e}$, а первый и последний слоги записи элемента $g_{2}-$ множеству $F_{f(1)} \backslash H_{f} \varphi_{+f}$;

$(b)$ для любого простого числа $r$ и для любого гомоморфизма $\rho$ подгруппы $S_{1}$ на конечную $r$-группу из соотношения $r \neq p$ следует, что $g_{1} \rho=1$; для любого простого числа $s$ и для любого гомоморфизма $\sigma$ подгруппы $S_{2}$ на конечную $s$-группу из соотношения $s \neq q$ следует, что $g_{2} \sigma=1$.

Покажем, что элемент $g=\left[g_{1}, g_{2}\right]$ отличен от 1 . Тогда по предложению 3 , применяемому к группе $F$, подгруппам $S_{1}, S_{2}$, элементам $g_{1}, g_{2}$ и числам $p, q$, получим, что $p=q$, как и требовалось.

Пусть $\widetilde{T}=(\widetilde{V}, \widetilde{E})$ - поддерево дерева $T$, представляющее собой путь, соединяющий вершины $e(-1)$ и $f(1), \widetilde{P}-$ соответствующее поддереву $\widetilde{T}$ древесное произведение. Если $e=f$, то $\widetilde{P}=P_{e}=P_{f}$ и в силу свойства $(a)$ элемент $g$ имеет в этой группе несократимую запись длины, не меньшей 12 . Согласно предложению 4 отсюда следует, что $g \neq 1$.

Пусть $e \neq f$, дерево $\widetilde{T}_{1}$ получается из $\widetilde{T}$ в результате удаления ребра $e$ и вершины $e(-1)$, дерево $\widetilde{T}_{2}-$ из $\widetilde{T}_{1}$ посредством удаления ребра $f$ и вершины $f(1)$. 
Пусть также $\widetilde{P}_{1}$ и $\widetilde{P}_{2}-$ древесные произведения, соответствующие деревьям $\widetilde{T}_{1}$ и $\widetilde{T}_{2}$. Тогда группа $\widetilde{P}$ является свободным произведением групп $P_{e}$ и $\widetilde{P}_{1}$ с объединенной подгруппой $F_{e(1)}$, а группа $\widetilde{P}_{1}-$ свободным произведением групп $\widetilde{P}_{2}$ и $P_{f}$ с объединенной подгруппой $F_{f(-1)}$.

Так как элемент $g_{1}$ имеет в группе $P_{e}$ несократимую запись неединичной длины, в силу предложения 4 он не лежит в свободном множителе $F_{e(1)}$. По тем же причинам $g_{2} \notin F_{f(-1)}$. Согласно предложению 5 в группе $\widetilde{P}_{1}$ справедливо равенство $\widetilde{P}_{2} \cap P_{f}=F_{f(-1)}$. Поскольку $F_{e(1)} \leq \widetilde{P}_{2}$, отсюда следует, что $g_{2} \notin F_{e(1)}$. Значит, элемент $g$ имеет в группе $\widetilde{P}$, рассматриваемой как обобщенное свободное произведение групп $P_{e}$ и $\widetilde{P}_{1}$, несократимую запись длины 4 и в силу предложения 4 отличен от 1.

Случай 2. $e \in E_{T}, f \notin E_{T}$ или $e \notin E_{T}, f \in E_{T}$. Пусть $P$ - древесное произведение, соответствующее максимальному поддереву $T$. Без потери общности можно считать, что $e \in E_{T}, f \notin E_{T}$. Поэтому согласно предложению 6 , применяемому к обобщенному свободному произведению

$$
P_{e}=\left\langle F_{e(1)} * F_{e(-1)} ; H_{e} \varphi_{+e}=H_{e} \varphi_{-e}\right\rangle
$$

и элементу $x_{e}$, а также предложению 8 , применяемому к HNN-расширению

$$
P(f)=\left\langle P, t_{f} ; t_{f}^{-1}\left(H_{f} \varphi_{+f}\right) t_{f}=H_{f} \varphi_{-f}\right\rangle
$$

и элементу $x_{f}$, существуют конечно порожденные подгруппы $S_{1} \leq P_{e}, S_{2} \leq P(f)$ и элементы $g_{1} \in S_{1}, g_{2} \in S_{2}$, обладающие следующими свойствами:

(a) элемент $g_{1}$ имеет в группе $P_{e}$ несократимую запись неединичной длины;

$(b)$ элемент $g_{2}$ имеет в группе $P(f)$ приведенную запись ненулевой длины, начинающуюся на $t_{f}^{-1}$ и заканчивающуюся на $t_{f}$;

$(c)$ для любого простого числа $r$ и для любого гомоморфизма $\rho$ подгруппы $S_{1}$ на конечную $r$-группу из соотношения $r \neq p$ следует, что $g_{1} \rho=1$; для любого простого числа $s$ и для любого гомоморфизма $\sigma$ подгруппы $S_{2}$ на конечную $s$-группу из соотношения $s \neq q$ следует, что $g_{2} \sigma=1$.

Пусть $T_{1}$ и $T_{2}$ - компоненты связности графа, получающегося из $T$ путем удаления ребра $e, P_{1}$ и $P_{2}-$ соответствующие древесные произведения. Тогда группа $P$ представляет собой древесное произведение групп $P_{1}, P_{e}$ и $P_{2}$ с объединенными подгруппами $F_{e(1)}$ и $F_{e(-1)}$. Из предложения 5 следует, что $P_{1} \cap P_{e} \subseteq F_{e(1)} \cup F_{e(-1)}$ и $P_{2} \cap P_{e} \subseteq F_{e(1)} \cup F_{e(-1)}$. Так как элемент $g_{1}$ имеет в группе $P_{e}$ несократимую запись неединичной длины, по предложению 4 $g_{1} \notin F_{e(1)} \cup F_{e(-1)}$. Отсюда вытекает, что $g_{1} \notin F_{v}$ для каждой вершины $v \in V$ и, в частности, $g_{1} \notin H_{f} \varphi_{-f}$. Поэтому запись элемента

$$
\left[g_{1}, g_{2}\right]=g_{1}^{-1} g_{2}^{-1} g_{1} g_{2} \in P(f)
$$

является приведенной, имеет ненулевую длину, и по предложению $7\left[g_{1}, g_{2}\right] \neq 1$. Как и в случае 1 , в силу предложения 3 отсюда следует, что $p=q$.

Случай 3. $e, f \notin E_{T}$. Пусть снова $P$ - древесное произведение, соответствующее максимальному поддереву $T$. Согласно предложению 8 , применяемому сначала к HNN-расширению

$$
P(e)=\left\langle P, t_{e} ; t_{e}^{-1}\left(H_{e} \varphi_{+e}\right) t_{e}=H_{e} \varphi_{-e}\right\rangle
$$


и элементу $x_{e}$, а затем к HNN-расширению

$$
P(f)=\left\langle P, t_{f} ; t_{f}^{-1}\left(H_{f} \varphi_{+f}\right) t_{f}=H_{f} \varphi_{-f}\right\rangle
$$

и элементу $x_{f}$, найдутся конечно порожденные подгруппы $S_{1} \leq P(e), S_{2} \leq P(f)$ и элементы $g_{1} \in S_{1}, g_{2} \in S_{2}$, обладающие следующими свойствами:

$(a)$ элементы $g_{1}$ и $g_{2}$ имеют в группах $P(e)$ и $P(f)$ соответственно приведенные записи ненулевой длины; при этом запись элемента $g_{1}$ начинается на $t_{e}^{-1}$ и заканчивается на $t_{e}$, а запись элемента $g_{2}$ начинается на $t_{f}^{-1}$ и заканчивается на $t_{f}$;

(b) для любого простого числа $r$ и для любого гомоморфизма $\rho$ подгруппы $S_{1}$ на конечную $r$-группу из соотношения $r \neq p$ следует, что $g_{1} \rho=1$; для любого простого числа $s$ и для любого гомоморфизма $\sigma$ подгруппы $S_{2}$ на конечную $s$-группу из соотношения $s \neq q$ следует, что $g_{2} \sigma=1$.

Пусть также $c \in F_{e(-1)} \backslash H_{e} \varphi_{-e}$ и

$$
g=\left[c^{-1} g_{1} c, g_{2}\right]=c^{-1} g_{1}^{-1} c g_{2}^{-1} c^{-1} g_{1} c g_{2} .
$$

Тогда в группе $F$, рассматриваемой как HNN-расширение группы $P$, запись элемента $g$ является приведенной и имеет ненулевую длину (это очевидно, если $e \neq f$, и вытекает из соотношения $c \in P \backslash H_{e} \varphi_{-e}$ в противном случае). Отсюда по предложению 7 следует, что $g \neq 1$. Применяя теперь предложение 3 к группе $F$, подгруппам $c^{-1} S_{1} c, S_{2}$, элементам $c^{-1} g_{1} c, g_{2}$ и числам $p, q$, получаем, что $p=q$.

\section{§6. Доказательство теоремы 1}

Если для любых $e \in E, \varepsilon= \pm 1$ подгруппа $H_{e} \varphi_{\varepsilon e}$ изолирована в группе $F_{e(\varepsilon)}$, то в качестве $p$ можно взять любое простое число. Поэтому далее будем считать, что для некоторых $e \in E, \varepsilon= \pm 1$ подгруппа $H_{e} \varphi_{\varepsilon e}$ не является изолированной в группе $F_{e(\varepsilon)}$. Тогда существуют элемент $x_{e} \in F_{e(\varepsilon)} \backslash H_{e} \varphi_{\varepsilon e}$ и простое число $p$ такие, что $x_{e}^{p} \in H_{e} \varphi_{\varepsilon e}$.

Предположим, что ребро $f \in E$, число $\delta= \pm 1$, элемент $x_{f} \in F_{f(\delta)} \backslash H_{f} \varphi_{\delta f}$ и простое число $q$ таковы, что $x_{f}^{q} \in H_{f} \varphi_{\delta f}$. Тогда по предложению $9 p=q$. Отсюда следует, что если для некоторых $f \in E$ и $\delta= \pm 1$ подгруппа $H_{f} \varphi_{\delta f}$ не является изолированной в группе $F_{f(\delta)}$, то она $p^{\prime}$-изолирована в ней. В частности, это верно и для подгруппы $H_{e} \varphi_{\varepsilon e}$.

\section{§ 7. Доказательство теоремы 2}

Если для любой вершины $v \in V$ имеет место соотношение $\left[F_{v}: H\right] \leq 2$, то подгруппа $H 2^{\prime}$-изолирована в группе $F_{v}$ для каждой $v \in V$. Поэтому далее будем считать, что существует вершина $v \in V$ такая, что $\left[F_{v}: H\right]>2$.

Производя необходимые переобозначения, не влияющие на представление группы $F$, можно считать, что все ребра дерева $G$ направлены от вершины $v$. Отсюда следует, в частности, что если два ребра инцидентны одной вершине, то последняя служит концом одного из них и началом другого.

Если $e \in E-$ некоторое ребро, то через $\varphi_{e}$ будем обозначать изоморфизм $\varphi_{+e}^{-1} \varphi_{-e}: H_{e} \varphi_{+e} \rightarrow H_{e} \varphi_{-e}$. Если ребра $e, f \in E$ таковы, что $e(-1)=f(1)$, то по определению конструкции свободного произведения с одной объединенной подгруппой $H_{e} \varphi_{-e}=H_{f} \varphi_{+f}$ и, значит, определена композиция изоморфизмов $\varphi_{e} \varphi_{f}$. 
Пусть $G^{\prime}$ - дерево-звезда с тем же множеством вершин, что и у дерева $G$, причем центром этой звезды является вершина $v$, из которой ведут ребра во все остальные вершины. Пусть также вершинам дерева $G^{\prime}$ соответствуют те же группы, что и вершинам дерева $G$. Если $e=(v, w)-$ ребро дерева $G^{\prime}$ и $e_{1}, e_{2}, \ldots, e_{n}-$ путь, ведущий в дереве $G$ из вершины $v$ в вершину $w$, то сопоставим ребру е группу $H_{e}=H_{e_{1}}$ и вложения $\varphi_{+e}=\varphi_{+e_{1}}$, $\varphi_{-e}=\varphi_{+e_{1}} \varphi_{e_{1}} \varphi_{e_{2}} \ldots \varphi_{e_{n}}$. В результате получим граф групп $\mathscr{G}^{\prime}$, причем для любого его ребра $e=(v, w)$ изоморфные вложения сопоставленных концам этого ребра групп $F_{v}$ и $F_{w}$ в группу $F$ отображают подгруппы $H_{e} \varphi_{+e}$ и $H_{e} \varphi_{-e}$ на $H$.

Покажем, что фундаментальные группы $F$ и $F^{\prime}$ графов $\mathscr{G}$ и $\mathscr{G}^{\prime}$ изоморфны. В самом деле, если, как и выше, $e=(v, w)$ - ребро дерева $G^{\prime}$ и $e_{1}, e_{2}, \ldots$, $e_{n}$ - путь, ведущий в дереве $G$ из вершины $v$ в вершину $w$, то из соотношений $h=h \varphi_{e_{i}}\left(i \in\{1, \ldots, n\}, h \in H_{e_{i}} \varphi_{+e_{i}}\right)$, справедливых в группе $F$, следует, что $h=h \varphi_{e_{1}} \varphi_{e_{2}} \ldots \varphi_{e_{n}}\left(h \in H_{e_{1}} \varphi_{+e_{1}}\right)$ и потому $h \varphi_{+e}=h \varphi_{-e}$ для каждого $h \in H_{e}$. Обратно, если $g=(u, w)-$ ребро дерева $G, e_{1}, e_{2}, \ldots, e_{n}=g-$ путь, ведущий в дереве $G$ из вершины $v$ в вершину $w, e=(v, w)$ и $f=(v, u)$ - ребра дерева $G^{\prime}$, то из соотношений $h \varphi_{+e}=h \varphi_{-e}\left(h \in H_{e}\right)$ и $h \varphi_{+f}=h \varphi_{-f}\left(h \in H_{f}\right)$ группы $F^{\prime}$ вытекает, что $h=h \varphi_{e_{1}} \varphi_{e_{2}} \ldots \varphi_{e_{n}}$ и $h=h \varphi_{e_{1}} \varphi_{e_{2}} \ldots \varphi_{e_{n-1}}\left(h \in H_{e_{1}} \varphi_{+e_{1}}\right)$, откуда $h=h \varphi_{e_{n}}$ для всех $h \in H_{e_{n}} \varphi_{+e_{n}}$ и потому $h \varphi_{+g}=h \varphi_{-g}$ для каждого $h \in H_{g}$. Поскольку остальные определяющие соотношения и все образующие в группах $F$ и $F^{\prime}$ одинаковы, требуемый изоморфизм имеет место.

Заметим, что если для некоторого ребра $e$ дерева $G^{\prime}$ справедливо равенство $H_{e} \varphi_{-e}=F_{e(-1)}$, то все определяющие соотношения группы $F_{e(-1)}$ выводимы из определяющих соотношений группы $F_{e}(1)$. Поэтому все образующие и определяющие соотношения группы $F_{e(-1)}$, а также всевозможные соотношения вида $h \varphi_{+e}=h \varphi_{-e}\left(h \in H_{e}\right)$ могут быть исключены из представления группы $F^{\prime}$. Эта операция равносильна удалению из графа групп $\mathscr{G}^{\prime}$ ребра $e$ вместе с вершиной $e(-1)$ и сопоставленными этим ребру и вершине группами.

Удалив из $\mathscr{G}^{\prime}$ все ребра описанного вида, получим граф групп $\mathscr{G}^{\prime \prime}$, фундаментальная группа $F^{\prime \prime}$ которого изоморфна $F$ и удовлетворяет условию теоремы 1. Следовательно, существует простое число $p$ такое, что для любого ребра e графа $\mathscr{G}^{\prime \prime}$ подгруппа $H_{e} \varphi_{+e} p^{\prime}$-изолирована в группе $F_{e(1)}=F_{v}$ и подгруппа $H_{e} \varphi-e p^{\prime}$-изолирована в группе $F_{e(-1)}$.

Пусть $w$ - произвольная вершина графа $\mathscr{G}$, отличная от $v$ и такая, что в группе $F$ имеет место соотношение $H \neq F_{w}$ (хотя бы одна такая вершина существует по условию теоремы). Тогда ребро $e=(v, w)$ графа $\mathscr{G}^{\prime}$ не было удалено при переходе к $\mathscr{G}^{\prime \prime}$, и так как вложения групп $F_{v}$ и $F_{w}$ в $F$ отображают подгруппы $H_{e} \varphi_{+e}$ и $H_{e} \varphi_{-e}$ на $H$, то подгруппа $H p^{\prime}$-изолирована в группах $F_{v}$ и $F_{w}$. Теорема доказана.

\section{§ 8. Доказательство теоремы 3}

Если для каждого $e \in E$ хотя бы один из индексов $\left[F_{v}: H_{e} \varphi_{+e}\right],\left[F_{v}: H_{e} \varphi_{-e}\right]$ больше двух, то требуемое утверждение вытекает из теоремы 1. Поэтому будем считать, что для некоторого $e \in E$ справедливы равенства $\left[F_{v}: H_{e} \varphi_{+e}\right]=2$ и $\left[F_{v}: H_{e} \varphi_{-e}\right]=2$.

Пусть $x_{e} \in F_{v} \backslash H_{e} \varphi_{+e}, y_{e} \in F_{v} \backslash H_{e} \varphi_{-e}$. Из соотношений $\left[F_{v}: H_{e} \varphi_{+e}\right]=$ $\left[F_{v}: H_{e} \varphi_{-e}\right]=2$ следует, что $x_{e}^{2} \in H_{e} \varphi_{+e}, t_{e}^{-1} x_{e}^{2} t_{e} \in H_{e} \varphi_{-e}$ и

$$
\left[H_{e} \varphi_{-e}: H_{e} \varphi_{+e} \cap H_{e} \varphi_{-e}\right] \leq 2 .
$$


Поэтому $t_{e}^{-1} x_{e}^{4} t_{e} \in H_{e} \varphi_{+e} \cap H_{e} \varphi_{-e}$ и $t_{e}^{-2} x_{e}^{4} t_{e}^{2} \in H_{e} \varphi_{-e}$. Значит, группа $U=$ $\operatorname{sgp}\left\{t_{e}^{-1} x_{e}^{2} t_{e}, t_{e}^{-2} x_{e}^{4} t_{e}^{2}, y\right\}$ является конечно порожденной подгруппой группы $F_{v}$ и потому удовлетворяет нетривиальному тождеству, которое в силу предложения 1 можно считать имеющим вид (3).

Положим

$$
\begin{aligned}
S_{1} & =\operatorname{sgp}\left\{x_{e}, y_{e}, t_{e}\right\} \\
g_{1} & =w\left(y_{e}, t_{e}^{-1} x_{e} t_{e}, t_{e}^{-2} x_{e} t_{e}^{2}\right) \\
& =w_{0}\left(t_{e}^{-1} x_{e} t_{e}, t_{e}^{-2} x_{e} t_{e}^{2}\right) y_{e}^{\varepsilon_{1}} w_{1}\left(t_{e}^{-1} x_{e} t_{e}, t_{e}^{-2} x_{e} t_{e}^{2}\right) \ldots y_{e}^{\varepsilon_{n}} w_{n}\left(t_{e}^{-1} x_{e} t_{e}, t_{e}^{-2} x_{e} t_{e}^{2}\right) .
\end{aligned}
$$

Согласно предложению 1 для каждого $r \in\{0, \ldots, n\}$

$$
w_{r}\left(t_{e}^{-1} x_{e} t_{e}, t_{e}^{-2} x_{e} t_{e}^{2}\right) \in\left\{t_{e}^{-1} x_{e}^{ \pm 1} t_{e}, t_{e}^{-2} x_{e}^{ \pm 1} t_{e}^{2},\left(t_{e}^{-1} x_{e} t_{e}^{-1} x_{e}^{-1} t_{e}^{2}\right)^{ \pm 1}\right\} .
$$

Поскольку $x_{e} \in F_{v} \backslash H_{e} \varphi_{+e}$ и $y_{e} \in F_{v} \backslash H_{e} \varphi_{-e}$, указанная выше запись элемента $g_{1}$ приведена.

Пусть $r$ - простое число, отличное от 2 , и $\rho-$ некоторый гомоморфизм подгруппы $S_{1}$ на конечную $r$-группу. Так как $(r, 4)=(r, 2)=1$, из включений $t_{e}^{-1} x_{e}^{2} t_{e} \in U, t_{e}^{-2} x_{e}^{4} t_{e}^{2} \in U$ и предложения 2 следует, что $\left(t_{e}^{-1} x_{e} t_{e}\right) \rho \in U \rho$ и $\left(t_{e}^{-2} x_{e} t_{e}^{2}\right) \rho \in U \rho$. Поскольку группа $U$ удовлетворяет тождеству (3), отсюда вытекает, что $g_{1} \rho=1$. Таким образом, подгруппа $S_{1}$ и элемент $g_{1}$ обладают следующими свойствами:

$\left(a_{1}\right)$ элемент $g_{1}$ имеет в группе $F$ приведенную запись ненулевой длины, начинающуюся на $t_{e}^{-1}$ и заканчивающуюся на $t_{e}$;

$\left(b_{1}\right)$ для любого простого числа $r$ и для любого гомоморфизма $\rho$ подгруппы $S_{1}$ на конечную $r$-группу из соотношения $r \neq 2$ следует, что $g_{1} \rho=1$.

Предположим, что ребро $f \in E \backslash\{e\}$, число $\delta= \pm 1$, элемент $x_{f} \in F_{v} \backslash H_{f} \varphi_{\delta f}$ и простое число $q$ таковы, что $x_{f}^{q} \in H_{f} \varphi_{\delta f}$ и хотя бы один из индексов $\left[F_{v}\right.$ : $\left.H_{f} \varphi_{+f}\right],\left[F_{v}: H_{f} \varphi_{-f}\right]$ больше двух. Тогда согласно предложению 8 , применяемому к HNN-расширению

$$
F_{v}(f)=\left\langle F_{v}, t_{f} ; t_{f}^{-1}\left(H_{f} \varphi_{+f}\right) t_{f}=H_{f} \varphi_{-f}\right\rangle
$$

и элементу $x_{f}$, найдутся конечно порожденная подгруппа $S_{2} \leq F_{v}(f)$ и элемент $g_{2} \in S_{2}$, обладающие следующими свойствами:

$\left(a_{2}\right)$ элемент $g_{2}$ имеет в группе $F_{v}(f)$ приведенную запись ненулевой длины, начинающуюся на $t_{f}^{-1}$ и заканчивающуюся на $t_{f}$;

$\left(b_{2}\right)$ для любого простого числа $s$ и для любого гомоморфизма $\sigma$ подгруппы $S_{2}$ на конечную $s$-группу из соотношения $s \neq q$ следует, что $g_{2} \sigma=1$.

Положим $g=\left[g_{1}, g_{2}\right]$. Так как $e \neq f$, запись элемента $g$ является приведенной в группе $F$ и имеет ненулевую длину. Следовательно, по предложению 7 $g \neq 1$ и по предложению $3 q=2$. Таким образом, все связанные подгруппы $\mathrm{HNN}$-расширения $F$ являются $2^{\prime}$-изолированными в группе $F_{v}$.

\section{$\S 9$. Пример}

Пусть $\Phi=\langle a, b\rangle-$ свободная группа ранга 2 , и для каждого $i \geq 1$ пусть $\gamma_{i} \Phi$ обозначает $i$-й член нижнего центрального ряда группы $\Phi$. Положим $N_{i}=$ 
$\Phi / \gamma_{i+1} \Phi$, выберем в подгруппе $\gamma_{i} \Phi / \gamma_{i+1} \Phi$ произвольный неединичный элемент $c_{i}$ и рассмотрим группу

$$
D=\left\langle N_{i}(i \geq 1) ;\left[N_{i}, N_{j}\right]=1, c_{i}=c_{j}(i \neq j)\right\rangle,
$$

представляющую собой фактор-группу прямого произведения групп $N_{i}(i \geq 1)$ по нормальному замыканию множества элементов вида $c_{i} c_{j}^{-1}(i \neq j)$. Нетрудно показать, что поскольку для каждого $i \geq 1$ элемент $c_{i}$ лежит в центре группы $N_{i}$ и порождает в ней бесконечную циклическую подгруппу, тождественное отображение образующих группы $N_{i}$ в группу $D$ при любом $i$ продолжаемо до изоморфного вложения и потому группу $N_{i}$ можно считать подгруппой в $D$ [15]. Все элементы $c_{i}$ переходят при этих вложениях в один и тот же элемент $c$ группы $D$.

Так как прямое произведение нильпотентных групп $N_{i}(i \geq 1)$ является, очевидно, локально нильпотентной группой, тем же свойством обладает и его фактор-группа $D$. В частности, она локально удовлетворяет нетривиальному тождеству и локально аппроксимируется нильпотентными группами.

Покажем, что группа $D$ не удовлетворяет никакому нетривиальному тождеству. Действительно, пусть $w\left(x_{1}, \ldots, x_{n}\right)$ - произвольное нетривиальное тождество. Поскольку группа $\Phi$ не удовлетворяет тождеству $w$, найдутся элементы $f_{1}, \ldots, f_{n} \in \Phi$ такие, что $w\left(f_{1}, \ldots, f_{n}\right) \neq 1$. Так как $\bigcap_{i \geq 1} \gamma_{i} \Phi=1$ [16], то $w\left(f_{1}, \ldots, f_{n}\right) \notin \gamma_{i+1} \Phi$ для некоторого $i \geq 1$ и потому $w\left(f_{1} \varepsilon_{i}, \ldots, f_{n} \varepsilon_{i}\right) \neq 1$, где $\varepsilon_{i}: \Phi \rightarrow N_{i}-$ естественный гомоморфизм. Следовательно, группа $N_{i}$ не удовлетворяет тождеству $w$ и, поскольку она инъективно вкладывается в группу $D$, последняя также не удовлетворяет $w$.

Покажем, что при каждом гомоморфизме группы $D$ на нильпотентную группу образ элемента $c$ оказывается равен 1 и, следовательно, группа $D$ не аппроксимируется нильпотентными группами. Пусть $\sigma$ - гомоморфизм группы $D$ на нильпотентную группу ступени $k, \sigma_{k+1}$ - ограничение гомоморфизма $\sigma$ на подгруппу $N_{k+1}$ и $\varepsilon_{k+1}: \Phi \rightarrow N_{k+1}-$ естественный гомоморфизм. Так как группа $\Phi \varepsilon_{k+1} \sigma_{k+1}$ имеет ступень нильпотентности не выше $k$, то $\gamma_{k+1} \Phi \leq$ $\operatorname{ker} \varepsilon_{k+1} \sigma_{k+1}$. Поскольку $c=c_{k+1} \in \gamma_{k+1} \Phi / \gamma_{k+2} \Phi$, отсюда следует, что $c \sigma=$ $c \sigma_{k+1}=1$, что и требовалось.

Благодарности. Автор выражает благодарность своему научному руководителю Е. В. Соколову за ряд ценных советов и замечаний.

\section{ЛИТЕРАТУРА}

1. Serre J.-P. Trees. Berlin: Springer-Verl., 1980.

2. Neumann H. Generalized free products with amalgamated subgroups // Amer. J. Math. 1948. V. 70, N 3. P. 590-625.

3. Азаров Д. Н., Иванова Е. А. К вопросу о нильпотентной аппроксимируемости свободного произведения с объединением локально нильпотентных групп // Науч. тр. Иван. гос. ун-та. Математика. 1999. № 2. С. 5-7.

4. Савельичева Н. C., Соколов E. В. Одно необходимое условие нильпотентной аппроксимируемости HNN-расширения нильпотентной группы // Вестн. Иван. гос. ун-та. Сер. Естеств., обществ. науки. 2015. № 1. С. 64-69.

5. Sokolov E. V. A necessary condition for the residual nilpotence of HNN-extensions // Lobachevskii J. Math. 2018. V. 39, N 2. P. 281-285.

6. Соколов E. В. Об отделимости подгрупп нильпотентных групп в классе конечных $\pi$-групп // Сиб. мат. журн. 2014. Т. 55, № 6. С. 1381-1390.

7. Соколов Е. В. Об отделимости подгрупп нильпотентно аппроксимируемых групп в классе конечных $\pi$-групп // Сиб. мат. журн. 2017. Т. 58, № 1. С. 219-229. 
8. Shirvani M. On residually finite HNN-extensions // Arch. Math. 1985. V. 44. P. 110-115.

9. Hirsch K. A. On infinite soluble groups (IV) // J. Lond. Math. Soc. 1952. V. 27. P. 81-85.

10. Холл Ф. Нильпотентные группы // Математика. 1968. Т. 12, № 1. С. 3-36.

11. Магнус B., Каррас А., Солитэр Д. Комбинаторная теория групп. М.: Наука, 1974.

12. Линдон P., Шупп П. Комбинаторная теория групп. М.: Мир, 1980.

13. Higman G., Neumann B. H., Neumann H. Embedding theorems for groups // J. Lond. Math. Soc. 1949. V. 24. P. 247-254.

14. Karras A., Solitar D. The subgroups of a free product of two groups with an amalgamated subgroups // Trans. Amer. Math. Soc. 1970. V. 150. P. 227-254.

15. Соколов E. В., Туманова Е. А. Обобщенные прямые произведения групп и их применение к изучению аппроксимируемости свободных конструкций групп // Междунар. конф. «Мальцевские чтения»: тез. докл. Новосибирск, 19-22 ноября 2018 г. Новосибирск, 2018. C. 117.

16. Magnus W. Beziehungen zwischen Gruppen und Idealen in einem speziellen Ring // Math. Ann. 1935. Bd 111. S. 259-280.

Поступила в редакцию 3 декабря 2018 г.

После доработки 3 декабря 2018 г.

Принята к публикации 15 мая 2019 г.

Куваев Александр Евгеньевич

Ивановский государственный университет, ул. Ермака, 39, Иваново 153025

alexander@kuvaev . me 